\title{
Simple Closed Analytic Formulas for the Approximation of the Legendre Complete Elliptic Integrals $K(k)$ and $E(k)$ (and their First Derivatives)
}

\author{
RICHARD SELESCU
}

Flow Physics Department, Experimental Aerodynamics Compartment, Trisonic Wind Tunnel Laboratory "Elie Carafoli" National Institute for Aerospace Research - INCAS (under the Aegis of the Romanian Academy) Bucharest, Sector 6, Bd. Iuliu Maniu, No. 220, Code 061126

ROMANIA

Abstract: Two sets of closed analytic functions are proposed for the approximate calculus of the complete elliptic integrals of the 1st and 2nd kind in the normal form due to Legendre, their expressions having a remarkable simplicity and accuracy. The special usefulness of the newly proposed original formulas consists in that they allow performing the analytic study of variation of the functions in which they appear, using derivatives (they being expressed in terms of elementary functions only, without any special function; this would mean replacing one difficulty by another of the same kind). Comparative tables of the approximate values so obtained and the exact ones, reproduced from special functions tables are given (vs. the elliptic integrals modulus k). It is to be noticed that both sets of formulas are given neither by spline nor by regression functions, but by asymptotic expansions, the identity with the exact functions being accomplished for the left domain's end. As for their simplicity, the formulas in $\mathrm{k}$ / k' do not need any mathematical table (are purely algebraic). As for their accuracy, the 2nd set, although more intricate, gives more accurate values than the 1st one and extends itself more closely to the right domain's end. Legendre complete elliptic integrals of the 1st and 2nd kinds are very useful in Signal Processing and especially in the design of Digital Filters ( Legendre Digital Filters).

Key-Words: analytic methods; Legendre complete elliptic integrals of the $1^{\text {st }}$ and $2^{\text {nd }}$ kind, $\mathrm{K}(k)$ and $\mathrm{E}(k)$; elliptic integral's modulus $k$; elliptic integral's complementary modulus $k$; tables of Legendre complete elliptic integrals; approximate formulas Digital Signal Processing Filters

Received: June 24, 2020. Revised: October 17, 2020. Accepted: November 26, 2020. Published: December 2 ,

\section{1 Introduction - elliptic integrals}

There are many interesting domains in pure and applied mathematics where appear one or both complete elliptic integrals of the $1^{\text {st }}$ and $2^{\text {nd }}$ kind in the normal form due to Legendre. The period of oscillations in a vacuum of the simple pendulum, in the dynamics of a constrained heavy particle, is given by a complete elliptic integral of the $1^{\text {st }}$ kind. The length of an ellipse, in the geometry of plane curves, as well as the lift coefficient of a thin delta wing with subsonic leading edges, in supersonic aerodynamics (small perturbations theory), are given by a complete elliptic integral of the $2^{\text {nd }}$ kind. The following relations define these integrals of the $1^{\text {st }}$ and $2^{\text {nd }}$ kind, respectively $\mathrm{K}(k)=\int_{0}^{\pi / 2}\left(1-k^{2} \sin ^{2} \varphi\right)^{-1 / 2} d \varphi=\int_{0}^{1}\left[\left(1-t^{2}\right)\left(1-k^{2} t^{2}\right)\right]^{-1 / 2} d t ;$ $\mathrm{E}(k)=\int_{0}^{\pi / 2}\left(1-k^{2} \sin ^{2} \varphi\right)^{1 / 2} d \varphi=\int_{0}^{1}\left[\left(1-k^{2} t^{2}\right)\left(1-t^{2}\right)^{-1}\right]^{1 / 2} d t ;$ $k=\sin \theta \geq 0$ is called modulus. $\mathrm{K}(k), \mathrm{E}(k)$ are typical elliptic integrals. They do not admit primitive functions (cannot be expressed in terms of elementary functions), being calculated by expanding the integrands into series, integrating term-by-term, and presented vs. $k \in[0,1]$, or vs. $\theta \in[0, \pi / 2]$, in some mathematical tables [1] - [6]. Modern mathematics defines an elliptic integral as any function $f$ which can be expressed in the form $f(x)=\int_{c}^{x} R\left[t, P(t)^{1 / 2}\right] d t ; R$ is a rational function of its two arguments; $P$ is a polynomial of degrees 3 or 4 with no repeated roots; $c$ is a constant. The values given in some special tables allow performing the calculus for a given case (point), but not the analytic study of variation of the functions in which these integrals appear, using the derivatives. In the next chapter two sets (subscripts 0 and 1 ) of closed analytic functions are given for the approximate calculus of $\mathrm{K}(k)$ and $\mathrm{E}(k)$. The method used in this work is purely analytic, not needing any numerical procedure, or sophisticated computer programs. There also is a Legendre complete elliptic integral of the $3^{\text {rd }}$ kind. Legendre complete elliptic integrals of the 1st and 2nd kinds are very useful in Signal Processing and especially in the design of Digital Filters ( Legendre Digital Filters)

\section{The two sets of newly proposed formulas}

The complementary modulus is $k^{\prime}=\left(1-k^{2}\right)^{1 / 2}=\cos \theta$.

For the first set the following formulas are proposed:

$$
\begin{aligned}
& \mathrm{K}_{0}(k)=\frac{\pi}{\sqrt[4]{1-k^{2}}}\left(1-\frac{1}{2 \sqrt{2}} \sqrt{\frac{1+\sqrt{1-k^{2}}}{\sqrt[4]{1-k^{2}}}}\right)=\pi\left(\frac{1}{\sqrt{k^{\prime}}}-\frac{1}{2 \sqrt{2}} \frac{\sqrt{1+k^{\prime}}}{k^{\prime 3 / 4}}\right), \\
& \mathrm{K}_{0}(\theta)=\frac{\pi}{\cos ^{1 / 2} \theta}\left(1-\frac{1}{2} \frac{\cos (\theta / 2)}{\cos ^{1 / 4} \theta}\right)=\pi\left(\frac{1}{\cos ^{1 / 2} \theta}-\frac{1}{2} \frac{\cos \theta / 2)}{\cos ^{3 / 4} \theta}\right) . \\
& \mathrm{E}_{0}(k)=\frac{\pi}{4} \sqrt[4]{1-k^{2}}\left(\frac{3}{2} \frac{1+\sqrt{1-k^{2}}}{\sqrt[4]{1-k^{2}}}-1\right)=\frac{\pi}{4}\left[\frac{3}{2}\left(1+k^{\prime}\right)-\sqrt{k^{\prime}}\right], \\
& \mathrm{E}_{0}(\theta)=\frac{\pi}{4} \cos ^{1 / 2} \theta\left(3 \frac{\cos ^{2}(\theta / 2)}{\cos ^{1 / 2} \theta}-1\right)=\frac{\pi}{4}\left(3 \cos ^{2} \frac{\theta}{2}-\sqrt{\cos \theta}\right) .
\end{aligned}
$$


Similarly, for the second set are proposed the formulas:

$$
\begin{aligned}
& \mathrm{K}_{1}(k)=\frac{\pi \sqrt{2}}{\sqrt{\left(1+k^{\prime}\right) \sqrt{k^{\prime}}}}\left(1-\frac{\sqrt[4]{2}}{4} \frac{1+\sqrt{k^{\prime}}}{\sqrt[4]{\left(1+k^{\prime}\right) \sqrt{k^{\prime}}}}\right), \\
& \mathrm{K}_{1}(\theta)=\frac{\pi}{\cos (\theta / 2) \cos ^{1 / 4} \theta}\left(1-\frac{1}{4} \frac{1+\cos ^{1 / 2} \theta}{\cos ^{1 / 2}(\theta / 2) \cos ^{1 / 8} \theta}\right) . \\
& \mathrm{E}_{1}(k)=\frac{\pi}{4}\left[\frac{3}{2}\left(1+\sqrt{k^{\prime}}\right)^{2}-\sqrt{2} \sqrt{1+k^{\prime}} \sqrt[4]{k^{\prime}}\right]-k^{\prime} \cdot \mathrm{K}_{1}(k), \\
& \mathrm{E}_{1}(\theta)=\frac{\pi}{4}\left[\frac{3}{2}(1+\sqrt{\cos \theta})^{2}-2 \cos \frac{\theta}{2} \sqrt[4]{\cos \theta}\right]-\cos \theta \cdot \mathrm{K}_{1}(\theta) .
\end{aligned}
$$

Table 1. Values of the functions $\mathrm{K}$ (part one)

$\begin{array}{rcccc}\theta\left({ }^{\circ}\right) & k=\sin \theta & \mathrm{K}(k) & \mathrm{K}_{0}(k) & \mathrm{K}_{1}(k) \\ 0 & 0.00000 & 1.5708 & 1.5708 & 1.5708 \\ 1 & 0.01745 & 1.5709 & 1.5709 & 1.5709 \\ 2 & 0.03490 & 1.5713 & 1.5713 & 1.5713 \\ 3 & 0.05234 & 1.5719 & 1.5719 & 1.5719 \\ 4 & 0.06976 & 1.5727 & 1.5727 & 1.5727 \\ 5 & 0.08716 & 1.5738 & 1.5738 & 1.5738 \\ 6 & 0.10453 & 1.5751 & 1.5751 & 1.5751 \\ 7 & 0.12187 & 1.5767 & 1.5767 & 1.5767 \\ 8 & 0.13917 & 1.5785 & 1.5785 & 1.5785 \\ 9 & 0.15643 & 1.5805 & 1.5805 & 1.5805 \\ 10 & 0.17365 & 1.5828 & 1.5828 & 1.5828 \\ 11 & 0.19081 & 1.5854 & 1.5854 & 1.5854 \\ 12 & 0.20791 & 1.5882 & 1.5882 & 1.5882 \\ 13 & 0.22495 & 1.5913 & 1.5913 & 1.5913 \\ 14 & 0.24192 & 1.5946 & 1.5946 & 1.5946 \\ 15 & 0.25882 & 1.5981 & 1.5981 & 1.5981 \\ 16 & 0.27564 & 1.6020 & 1.6020 & 1.6020 \\ 17 & 0.29237 & 1.6061 & 1.6061 & 1.6061 \\ 18 & 0.30902 & 1.6105 & 1.6105 & 1.6105 \\ 19 & 0.32557 & 1.6151 & 1.6151 & 1.6151 \\ 20 & 0.34202 & 1.6200 & 1.6200 & 1.6200 \\ 21 & 0.35837 & 1.6252 & 1.6252 & 1.6252 \\ 22 & 0.37461 & 1.6307 & 1.6307 & 1.6307 \\ 23 & 0.39073 & 1.6365 & 1.6365 & 1.6365 \\ 24 & 0.40674 & 1.6426 & 1.6426 & 1.6426 \\ 25 & 0.42262 & 1.6490 & 1.6490 & 1.6490 \\ 26 & 0.43837 & 1.6557 & 1.6557 & 1.6557 \\ 27 & 0.45399 & 1.6627 & 1.6627 & 1.6627 \\ 28 & 0.46947 & 1.6701 & 1.6701 & 1.6701 \\ 29 & 0.48481 & 1.6777 & 1.6777 & 1.6777 \\ 30 & 0.50000 & 1.6858 & 1.6857 & 1.6858 \\ 31 & 0.51504 & 1.6941 & 1.6941 & 1.6941 \\ 32 & 0.52992 & 1.7028 & 1.7028 & 1.7028 \\ 33 & 0.54464 & 1.7119 & 1.7119 & 1.7119 \\ 34 & 0.55919 & 1.7214 & 1.7214 & 1.7214 \\ 35 & 0.57358 & 1.7312 & 1.7312 & 1.7312 \\ 36 & 0.58779 & 1.7415 & 1.7415 & 1.7415 \\ 37 & 0.60182 & 1.7522 & 1.7522 & 1.7522 \\ 38 & 0.61566 & 1.7633 & 1.7632 & 1.7633 \\ 39 & 0.62932 & 1.7748 & 1.7748 & 1.7748 \\ 40 & 0.64279 & 1.7868 & 1.7867 & 1.7868\end{array}$

$\begin{array}{ccccc}41 & 0.65606 & 1.7992 & 1.7992 & 1.7992 \\ 42 & 0.66913 & 1.8122 & 1.8121 & 1.8122 \\ 43 & 0.68200 & 1.8256 & 1.8256 & 1.8256 \\ 44 & 0.69466 & 1.8396 & 1.8395 & 1.8396 \\ 45 & 0.70711 & 1.8541 & 1.8540 & 1.8541 \\ 46 & 0.71934 & 1.8691 & 1.8691 & 1.8691 \\ 47 & 0.73135 & 1.8848 & 1.8847 & 1.8848 \\ 48 & 0.74314 & 1.9011 & 1.9009 & 1.9011 \\ 49 & 0.75471 & 1.9180 & 1.9178 & 1.9180 \\ 50 & 0.76604 & 1.9356 & 1.9354 & 1.9356 \\ 51 & 0.77715 & 1.9539 & 1.9536 & 1.9539 \\ 52 & 0.78801 & 1.9729 & 1.9726 & 1.9729 \\ 53 & 0.79864 & 1.9927 & 1.9923 & 1.9927 \\ 54 & 0.80902 & 2.0133 & 2.0128 & 2.0133 \\ 55 & 0.81915 & 2.0347 & 2.0341 & 2.0347 \\ 56 & 0.82904 & 2.0571 & 2.0564 & 2.0571 \\ 57 & 0.83867 & 2.0804 & 2.0795 & 2.0804 \\ 58 & 0.84805 & 2.1047 & 2.1037 & 2.1047 \\ 59 & 0.85717 & 2.1300 & 2.1288 & 2.1300 \\ 60 & 0.86603 & 2.1565 & 2.1551 & 2.1565 \\ 61 & 0.87462 & 2.1842 & 2.1825 & 2.1842 \\ 62 & 0.88295 & 2.2132 & 2.2111 & 2.2132 \\ 63 & 0.89101 & 2.2435 & 2.2410 & 2.2435 \\ 64 & 0.89879 & 2.2754 & 2.2723 & 2.2754 \\ 65 & 0.90631 & 2.3088 & 2.3051 & 2.3088 \\ 66 & 0.91355 & 2.3439 & 2.3394 & 2.3439 \\ 67 & 0.92050 & 2.3809 & 2.3754 & 2.3809 \\ 68 & 0.92718 & 2.4198 & 2.4132 & 2.4198 \\ 69 & 0.93358 & 2.4610 & 2.4530 & 2.4610 \\ 70 & 0.93969 & 2.5046 & 2.4948 & 2.5045 \\ 70.5 & 0.94264 & 2.5273 & 2.5165 & 2.5273 \\ 71 & 0.94552 & 2.5507 & 2.5389 & 2.5507 \\ 71.5 & 0.94832 & 2.5749 & & 2.5749 \\ 72 & 0.95106 & 2.5998 & & 2.5998 \\ 72.5 & 0.95372 & 2.6256 & & 2.6255 \\ 73 & 0.95630 & 2.6521 & & 2.6521 \\ 73.5 & 0.95882 & 2.6796 & & 2.6796 \\ 74 & 0.96126 & 2.7081 & & 2.7081 \\ 74.5 & 0.96363 & 2.7375 & & 2.7375 \\ 75 & 0.96593 & 2.7681 & & 2.7680 \\ 75.5 & 0.96815 & 2.7998 & & 2.7997 \\ 76 & 0.97030 & 2.8327 & & 2.8326 \\ 76.5 & 0.97237 & 2.8669 & & 2.8669 \\ 77 & 0.97437 & 2.9026 & & 2.9025 \\ 77.5 & 0.97630 & 2.9397 & & 2.9397 \\ 78 & 0.97815 & 2.9786 & & 2.9785 \\ 78.5 & 0.97992 & 3.0192 & & 3.0191 \\ 79 & 0.98163 & 3.0617 & & 3.0616 \\ 79.5 & 0.98325 & 3.1064 & & 3.1063 \\ 80 & 0.98481 & 3.1534 & & 3.1533 \\ 80.2 & 0.98541 & 3.1729 & & 3.1727 \\ 80.4 & 0.98600 & 3.1928 & & 3.1927 \\ 80.6 & 0.98657 & 3.2132 & & 3.2130 \\ 80.8 & 0.98714 & 3.2340 & & 3.2338 \\ 81 & 0.98769 & 3.2553 & & 3.2551\end{array}$


Table 1. Values of the functions K (part two)

\begin{tabular}{|c|c|c|c|}
\hline 81.2 & 0.98823 & 3.2771 & 3.2769 \\
\hline 81.4 & 0.98876 & 3.2995 & 3.2992 \\
\hline 81.6 & 0.98927 & 3.3223 & 3.3221 \\
\hline 81.8 & 0.98978 & 3.3458 & 3.3455 \\
\hline 82 & 0.99027 & 3.3699 & 3.3696 \\
\hline 82.2 & 0.99075 & 3.3946 & 3.3942 \\
\hline 82.4 & 0.99122 & 3.4199 & 3.4196 \\
\hline 82.6 & 0.99167 & 3.4460 & 3.4456 \\
\hline 82.8 & 0.99211 & 3.4728 & 3.4724 \\
\hline 83 & 0.99255 & 3.5004 & 3.4999 \\
\hline 83.2 & 0.99297 & 3.5288 & 3.5283 \\
\hline 83.4 & 0.99337 & 3.5581 & 3.5575 \\
\hline 83.6 & 0.99377 & 3.5884 & 3.5877 \\
\hline 83.8 & 0.99415 & 3.6196 & 3.6188 \\
\hline 84 & 0.99452 & 3.6519 & 3.6510 \\
\hline 84.2 & 0.99488 & 3.6852 & 3.6843 \\
\hline 84.4 & 0.99523 & 3.7198 & 3.7187 \\
\hline 84.6 & 0.99556 & 3.7557 & 3.7545 \\
\hline 84.8 & 0.99588 & 3.7930 & 3.7916 \\
\hline 85 & 0.99619 & 3.8317 & 3.8302 \\
\hline 85.2 & 0.99649 & 3.8721 & 3.8704 \\
\hline 85.4 & 0.99678 & 3.9142 & 3.9122 \\
\hline 85.6 & 0.99705 & 3.9583 & 3.9560 \\
\hline 85.8 & 0.99731 & 4.0044 & 4.0018 \\
\hline 86 & 0.99756 & 4.0528 & 4.0498 \\
\hline 86.2 & 0.99780 & 4.1037 & 4.1003 \\
\hline 86.4 & 0.99803 & 4.1574 & 4.1535 \\
\hline 86.6 & 0.99824 & 4.2142 & 4.2097 \\
\hline 86.8 & 0.99844 & 4.2744 & 4.2692 \\
\hline 87 & 0.99863 & 4.3387 & 4.3325 \\
\hline 87.2 & 0.99881 & 4.4073 & 4.4001 \\
\hline 87.4 & 0.99897 & 4.4811 & 4.4726 \\
\hline 87.6 & 0.99912 & 4.5609 & 4.5507 \\
\hline 87.8 & 0.99926 & 4.6477 & 4.6354 \\
\hline 88 & 0.99939 & 4.7427 & 4.7277 \\
\hline 88.2 & 0.99951 & 4.8478 & 4.8293 \\
\hline 88.4 & 0.99961 & 4.9654 & \\
\hline 88.6 & 0.99970 & 5.0988 & \\
\hline 88.8 & 0.99978 & 5.2527 & \\
\hline 89 & 0.99985 & 5.4329 & \\
\hline 89.1 & 0.99988 & 5.5402 & \\
\hline 89.2 & 0.99990 & 5.6579 & \\
\hline 89.3 & 0.99993 & 5.7914 & \\
\hline 89.4 & 0.99995 & 5.9455 & \\
\hline 89.5 & 0.99996 & 6.1278 & \\
\hline 89.6 & 0.99998 & 6.3509 & \\
\hline 89.7 & 0.99999 & 6.6385 & \\
\hline 89.8 & 0.99999 & 7.0440 & \\
\hline 89.9 & 1.00000 & 7.7371 & \\
\hline 90 & 1.00000 & $\infty$ & \\
\hline
\end{tabular}

The values strings in the last two columns of table 1 were canceled when each of the two closed analytic formulas proposed for the approximation of the Legendre complete elliptic integral of the $1^{\text {st }}$ kind $\mathrm{K}(k)$ gives too great relative errors ( $\geq 4 \%$ - also see chapter 3$)$ for being still accepted in the usual mathematical / technical calculus. The same procedure will be applied in case of the next table (no. 2), for the same reason, concerning the accuracy of the values given by each of the other two closed analytic formulas proposed for the approximation of the Legendre complete elliptic integral of the $2^{\text {nd }}$ kind $\mathrm{E}(k)$. The accuracy analysis of the two sets of formulas will be performed in the next chapter (no. 3). In chapter 4 some series representations for the exact functions and for both sets of approximation, as well as for their first order derivatives, will be given.

Table 2. Values of the functions $\mathrm{E}$ (part one)

$\begin{array}{ccccc}\theta\left({ }^{\circ}\right) & k=\sin \theta & \mathrm{E}(k) & \mathrm{E}_{0}(k) & \mathrm{E}_{1}(k) \\ 0 & 0.00000 & 1.5708 & 1.5708 & 1.5708 \\ 1 & 0.01745 & 1.5707 & 1.5707 & 1.5707 \\ 2 & 0.03490 & 1.5703 & 1.5703 & 1.5703 \\ 3 & 0.05234 & 1.5697 & 1.5697 & 1.5697 \\ 4 & 0.06976 & 1.5689 & 1.5689 & 1.5689 \\ 5 & 0.08716 & 1.5678 & 1.5678 & 1.5678 \\ 6 & 0.10453 & 1.5665 & 1.5665 & 1.5665 \\ 7 & 0.12187 & 1.5649 & 1.5649 & 1.5649 \\ 8 & 0.13917 & 1.5632 & 1.5632 & 1.5632 \\ 9 & 0.15643 & 1.5611 & 1.5611 & 1.5611 \\ 10 & 0.17365 & 1.5589 & 1.5589 & 1.5589 \\ 11 & 0.19081 & 1.5564 & 1.5564 & 1.5564 \\ 12 & 0.20791 & 1.5537 & 1.5537 & 1.5537 \\ 13 & 0.22495 & 1.5507 & 1.5507 & 1.5507 \\ 14 & 0.24192 & 1.5476 & 1.5476 & 1.5476 \\ 15 & 0.25882 & 1.5442 & 1.5442 & 1.5442 \\ 16 & 0.27564 & 1.5405 & 1.5405 & 1.5405 \\ 17 & 0.29237 & 1.5367 & 1.5367 & 1.5367 \\ 18 & 0.30902 & 1.5326 & 1.5326 & 1.5326 \\ 19 & 0.32557 & 1.5283 & 1.5283 & 1.5283 \\ 20 & 0.34202 & 1.5238 & 1.5238 & 1.5238 \\ 21 & 0.35837 & 1.5191 & 1.5191 & 1.5191 \\ 22 & 0.37461 & 1.5141 & 1.5141 & 1.5141 \\ 23 & 0.39073 & 1.5090 & 1.5090 & 1.5090 \\ 24 & 0.40674 & 1.5037 & 1.5037 & 1.5037 \\ 25 & 0.42262 & 1.4981 & 1.4981 & 1.4981 \\ 26 & 0.43837 & 1.4924 & 1.4924 & 1.4924 \\ 27 & 0.45399 & 1.4864 & 1.4864 & 1.4864 \\ 28 & 0.46947 & 1.4803 & 1.4803 & 1.4803 \\ 29 & 0.48481 & 1.4740 & 1.4740 & 1.4740 \\ 30 & 0.50000 & 1.4675 & 1.4675 & 1.4675 \\ 31 & 0.51504 & 1.4608 & 1.4608 & 1.4608 \\ 32 & 0.52992 & 1.4539 & 1.4539 & 1.4539 \\ 33 & 0.54464 & 1.4469 & 1.4469 & 1.4469 \\ 34 & 0.55919 & 1.4397 & 1.4397 & 1.4397 \\ 35 & 0.57358 & 1.4323 & 1.4323 & 1.4323 \\ 36 & 0.58779 & 1.4248 & 1.4248 & 1.4248 \\ 37 & 0.60182 & 1.4171 & 1.4171 & 1.4171 \\ 38 & 0.61566 & 1.4092 & 1.4093 & 1.4092 \\ 39 & 0.62932 & 1.4013 & 1.4013 & 1.4013 \\ 40 & 0.64279 & 1.3931 & 1.3932 & 1.3931\end{array}$


Table 2. Values of the functions E (part two)

$\begin{array}{ccccc}41 & 0.65606 & 1.3849 & 1.3849 & 1.3849 \\ 42 & 0.66913 & 1.3765 & 1.3765 & 1.3765 \\ 43 & 0.68200 & 1.3680 & 1.3680 & 1.3680 \\ 44 & 0.69466 & 1.3594 & 1.3594 & 1.3594 \\ 45 & 0.70711 & 1.3506 & 1.3507 & 1.3506 \\ 46 & 0.71934 & 1.3418 & 1.3419 & 1.3418 \\ 47 & 0.73135 & 1.3329 & 1.3330 & 1.3329 \\ 48 & 0.74314 & 1.3238 & 1.3239 & 1.3238 \\ 49 & 0.75471 & 1.3147 & 1.3148 & 1.3147 \\ 50 & 0.76604 & 1.3055 & 1.3057 & 1.3055 \\ 51 & 0.77715 & 1.2963 & 1.2964 & 1.2963 \\ 52 & 0.78801 & 1.2870 & 1.2872 & 1.2870 \\ 53 & 0.79864 & 1.2776 & 1.2778 & 1.2776 \\ 54 & 0.80902 & 1.2681 & 1.2684 & 1.2681 \\ 55 & 0.81915 & 1.2587 & 1.2590 & 1.2587 \\ 56 & 0.82904 & 1.2492 & 1.2496 & 1.2492 \\ 57 & 0.83867 & 1.2397 & 1.2401 & 1.2397 \\ 58 & 0.84805 & 1.2301 & 1.2307 & 1.2301 \\ 59 & 0.85717 & 1.2206 & 1.2212 & 1.2206 \\ 60 & 0.86603 & 1.2111 & 1.2118 & 1.2111 \\ 61 & 0.87462 & 1.2015 & 1.2024 & 1.2015 \\ 62 & 0.88295 & 1.1920 & 1.1930 & 1.1920 \\ 63 & 0.89101 & 1.1826 & 1.1838 & 1.1826 \\ 64 & 0.89879 & 1.1732 & 1.1745 & 1.1732 \\ 65 & 0.90631 & 1.1638 & 1.1654 & 1.1638 \\ 66 & 0.91355 & 1.1545 & 1.1564 & 1.1545 \\ 67 & 0.92050 & 1.1453 & 1.1475 & 1.1453 \\ 68 & 0.92718 & 1.1362 & 1.1387 & 1.1362 \\ 69 & 0.93358 & 1.1272 & 1.1301 & 1.1273 \\ 70 & 0.93969 & 1.1184 & 1.1217 & 1.1184 \\ 70.5 & 0.94264 & 1.1140 & 1.1176 & 1.1140 \\ 71 & 0.94552 & 1.1096 & 1.1135 & 1.1096 \\ 71.5 & 0.94832 & 1.1053 & & 1.1053 \\ 72 & 0.95106 & 1.1011 & & 1.1011 \\ 72.5 & 0.95372 & 1.0968 & & 1.0968 \\ 73 & 0.95630 & 1.0927 & & 1.0927 \\ 73.5 & 0.95882 & 1.0885 & & 1.0885 \\ 74 & 0.96126 & 1.0844 & & 1.0844 \\ 74.5 & 0.96363 & 1.0804 & & 1.0804 \\ 75 & 0.96593 & 1.0764 & & 1.0764 \\ 75.5 & 0.96815 & 1.0725 & & 1.0725 \\ 76 & 0.97030 & 1.0686 & & 1.0686 \\ 76.5 & 0.97237 & 1.0648 & & 1.0648 \\ 77 & 0.97437 & 1.0611 & & 1.0611 \\ 77.5 & 0.97630 & 1.0574 & & 1.0574 \\ 78 & 0.97815 & 1.0538 & & 1.0538 \\ 78.5 & 0.97992 & 1.0502 & & 1.0503 \\ 79 & 0.98163 & 1.0468 & & 1.0468 \\ 79.5 & 0.98325 & 1.0434 & & 1.0435 \\ 80 & 0.98481 & 1.0401 & & 1.0402 \\ 80.2 & 0.98541 & 1.0388 & & 1.0389 \\ 80.4 & 0.98600 & 1.0375 & & 1.0376 \\ 80.6 & 0.98657 & 1.0363 & & 1.0364 \\ 80.8 & 0.98714 & 1.0350 & & 1.0351\end{array}$

\begin{tabular}{|c|c|c|c|}
\hline 81 & 0.98769 & 1.0338 & 1.0339 \\
\hline 81.2 & 0.98823 & 1.0326 & 1.0327 \\
\hline 81.4 & 0.98876 & 1.0314 & 1.0315 \\
\hline 81.6 & 0.98927 & 1.0302 & 1.0303 \\
\hline 81.8 & 0.98978 & 1.0290 & 1.0292 \\
\hline 82 & 0.99027 & 1.0278 & 1.0280 \\
\hline 82.2 & 0.99075 & 1.0267 & 1.0269 \\
\hline 82.4 & 0.99122 & 1.0256 & 1.0258 \\
\hline 82.6 & 0.99167 & 1.0245 & 1.0247 \\
\hline 82.8 & 0.99211 & 1.0234 & 1.0236 \\
\hline 83 & 0.99255 & 1.0223 & 1.0226 \\
\hline 83.2 & 0.99297 & 1.0213 & 1.0215 \\
\hline 83.4 & 0.99337 & 1.0202 & 1.0205 \\
\hline 83.6 & 0.99377 & 1.0192 & 1.0196 \\
\hline 83.8 & 0.99415 & 1.0182 & 1.0186 \\
\hline 84 & 0.99452 & 1.0172 & 1.0176 \\
\hline 84.2 & 0.99488 & 1.0163 & 1.0167 \\
\hline 84.4 & 0.99523 & 1.0153 & 1.0158 \\
\hline 84.6 & 0.99556 & 1.0144 & 1.0150 \\
\hline 84.8 & 0.99588 & 1.0135 & 1.0141 \\
\hline 85 & 0.99619 & 1.0127 & 1.0133 \\
\hline 85.2 & 0.99649 & 1.0118 & 1.0125 \\
\hline 85.4 & 0.99678 & 1.0110 & 1.0118 \\
\hline 85.6 & 0.99705 & 1.0102 & 1.0110 \\
\hline 85.8 & 0.99731 & 1.0094 & 1.0103 \\
\hline 86 & 0.99756 & 1.0086 & 1.0097 \\
\hline 86.2 & 0.99780 & 1.0079 & 1.0091 \\
\hline 86.4 & 0.99803 & 1.0072 & 1.0085 \\
\hline 86.6 & 0.99824 & 1.0065 & 1.0080 \\
\hline 86.8 & 0.99844 & 1.0059 & 1.0075 \\
\hline 87 & 0.99863 & 1.0053 & 1.0071 \\
\hline 87.2 & 0.99881 & 1.0047 & 1.0067 \\
\hline 87.4 & 0.99897 & 1.0041 & 1.0064 \\
\hline 87.6 & 0.99912 & 1.0036 & 1.0062 \\
\hline 87.8 & 0.99926 & 1.0031 & 1.0060 \\
\hline 88 & 0.99939 & 1.0026 & 1.0060 \\
\hline 88.2 & 0.99951 & 1.0021 & 1.0061 \\
\hline 88.4 & 0.99961 & 1.0017 & \\
\hline 88.6 & 0.99970 & 1.0014 & \\
\hline 88.8 & 0.99978 & 1.0010 & \\
\hline 89 & 0.99985 & 1.0008 & \\
\hline 89.1 & 0.99988 & 1.0006 & \\
\hline 89.2 & 0.99990 & 1.0005 & \\
\hline 89.3 & 0.99993 & 1.0004 & \\
\hline 89.4 & 0.99995 & 1.0003 & \\
\hline 89.5 & 0.99996 & 1.0002 & \\
\hline 89.6 & 0.99998 & 1.0001 & \\
\hline 89.7 & 0.99999 & 1.0001 & \\
\hline 89.8 & 0.99999 & 1.0000 & \\
\hline 89.9 & 1.00000 & 1.0000 & \\
\hline 90 & 1.00000 & 1.0000 & \\
\hline
\end{tabular}

In the comparative tables 1 and 2, the $4 D$ (four digit) exact values of both Legendre complete elliptic integrals reproduced from special functions tables [6], as well as their $4 D$ approximate values obtained by applying the two 
sets of proposed closed analytic formulas were given (all versus the respective elliptic integrals modulus, $k=\sin \theta$ ). It is to be noticed that both sets of approximate formulas are not given by spline or regression functions, but by asymptotic expansions, the respective expressions having a remarkable simplicity (see, e.g.: the $2^{\text {nd }}$ form of $\mathrm{E}_{0}(k)$ or $\mathrm{E}_{0}(\theta)$; more, all newly found formulas in $k / k^{\prime}$ do not need any mathematical table, being purely algebraic) and accuracy (see table 3 ). The identity with the exact functions is satisfied for the left end $k=0\left(\theta=0^{\circ}\right)$ of the domain. As one can see, the $2^{\text {nd }}$ set of functions $\left(\mathrm{K}_{1}, \mathrm{E}_{1}\right)$, although something more intricate, gives more accurate values than the first one $\left(\mathrm{K}_{0}, \mathrm{E}_{0}\right)$ and extends itself more closely to the right end $k=1\left(\theta=90^{\circ}\right)$ of the domain.

\section{The accuracy of the two sets of formulas}

Let us define the following relative error functions: $\varepsilon_{\mathrm{K} 0}(k)=\mathrm{K}_{0}(k) / \mathrm{K}(k)-1 ; \quad \varepsilon_{\mathrm{K}_{1}}(k)=\mathrm{K}_{1}(k) / \mathrm{K}(k)-1$, for both sets of approximation of the $1^{\text {st }}$ kind integral and $\varepsilon_{\mathrm{E}_{0}}(k)=\mathrm{E}_{0}(k) / \mathrm{E}(k)-1 ; \quad \varepsilon_{\mathrm{E}_{1}}(k)=\mathrm{E}_{1}(k) / \mathrm{E}(k)-1$, for both sets of approximation of the $2^{\text {nd }}$ kind integral. Their values are given in the table 3 , being expressed in thousandths (\%o). These errors were calculated for the $1^{\text {st }}$ set $\left(\mathrm{K}_{0}\right.$ and $\left.\mathrm{E}_{0}\right)$ only in the field $\theta \in\left[54^{\circ}, 71^{\circ}\right]$ of the domain, with an increment of $1^{\circ}$, while for the $2^{\text {nd }}$ set $\left(\mathrm{K}_{1}\right.$ and $\left.\mathrm{E}_{1}\right)$ only in the field $\theta \in\left[84^{\circ} .8,88^{\circ} .2\right]$, with an increment of $0^{\circ} .2$, like in the above tables 1 and 2 .

Table 3. Relative errors $\varepsilon$ distribution

\begin{tabular}{|c|c|c|c|c|}
\hline$\theta\left(^{\circ}\right)$ & $k=\sin \theta$ & $\varepsilon_{\mathrm{K}_{0}}(\% 0)$ & $\varepsilon_{\mathrm{K}_{1}}(\%)$ & $\varepsilon_{\mathrm{E}_{0}}(\% o)$ \\
\hline 54 & 0.80902 & -0.250 & & +0.255 \\
\hline 55 & 0.81915 & -0.272 & & +0.243 \\
\hline 56 & 0.82904 & -0.353 & & +0.293 \\
\hline 57 & 0.83867 & -0.420 & & +0.334 \\
\hline 58 & 0.84805 & -0.497 & & +0.454 \\
\hline 59 & 0.85717 & -0.558 & & +0.502 \\
\hline 60 & 0.86603 & -0.669 & & +0.566 \\
\hline 61 & 0.87462 & -0.799 & & +0.742 \\
\hline 62 & 0.88295 & -0.961 & & +0.874 \\
\hline 63 & 0.89101 & -1.118 & & +0.973 \\
\hline 64 & 0.89879 & -1.366 & & +1.135 \\
\hline 65 & 0.90631 & -1.619 & & +1.377 \\
\hline 66 & 0.91355 & -1.918 & & +1.627 \\
\hline 67 & 0.92050 & -2.299 & & +1.900 \\
\hline 68 & 0.92718 & -2.709 & & +2.215 \\
\hline 69 & 0.93358 & -3.253 & & +2.573 \\
\hline 70 & 0.93969 & -3.907 & & +2.959 \\
\hline 71 & 0.94552 & -4.642 & & +3.525 \\
\hline
\end{tabular}

$\begin{array}{cccccc}84.8 & 0.99588 & - & -0.369 & - & +0.607 \\ 85 & 0.99619 & - & -0.396 & - & +0.592 \\ 85.2 & 0.99649 & - & -0.451 & - & +0.705 \\ 85.4 & 0.99678 & - & -0.500 & - & +0.748\end{array}$

$\begin{array}{clllll}85.6 & 0.99705 & - & -0.582 & - & +0.823 \\ 85.8 & 0.99731 & - & -0.652 & - & +0.932 \\ 86 & 0.99756 & - & -0.737 & - & +1.076 \\ 86.2 & 0.99780 & - & -0.832 & - & +1.160 \\ 86.4 & 0.99803 & - & -0.945 & - & +1.284 \\ 86.6 & 0.99824 & - & -1.077 & - & +1.453 \\ 86.8 & 0.99844 & - & -1.214 & - & +1.571 \\ 87 & 0.99863 & - & -1.421 & - & +1.743 \\ 87.2 & 0.99881 & - & -1.626 & - & +1.976 \\ 87.4 & 0.99897 & - & -1.894 & - & +2.275 \\ 87.6 & 0.99912 & - & -2.234 & - & +2.553 \\ 87.8 & 0.99926 & - & -2.655 & - & +2.922 \\ 88 & 0.99939 & - & -3.156 & - & +3.397 \\ 88.2 & 0.99951 & - & -3.808 & - & +4.004\end{array}$

The relative errors strings are stopped for values $\geq 4 \%$.

\section{Comparative series representations}

Expanding into power series, one obtains for the complete elliptic integrals the set of representations below ([5]- [7]):

$$
\begin{aligned}
& \mathrm{K}(k)=\frac{\pi}{2}\left(1+\frac{1}{4} k^{2}+\frac{9}{64} k^{4}+\frac{25}{256} k^{6}+\frac{1225}{16384} k^{8}+\frac{3969}{65536} k^{10}\right. \\
& \left.+\frac{53361}{1048576} k^{12}+\frac{184041}{4194304} k^{14}+\frac{41409225}{1073741824} k^{16}+\ldots\right) \\
& =\frac{\pi}{2}\left\{1+\sum_{n=1}^{\infty}\left[\frac{1 \cdot 3 \cdot \ldots(2 n-1)}{2 \cdot 4 \cdot \ldots \cdot 2 n}\right]^{2} k^{2 n}\right\}=\frac{\pi}{2}\left\{1+\sum_{n=1}^{\infty}\left[\frac{(2 n-1) ! !}{2^{n} n !}\right]^{2} k^{2 n}\right\} \\
& \mathrm{E}(k)=\frac{\pi}{2}\left(1-\frac{1}{4} k^{2}-\frac{3}{64} k^{4}-\frac{5}{256} k^{6}-\frac{175}{16384} k^{8}-\frac{441}{65536} k^{10}\right. \\
& \left.-\frac{4851}{1048576} k^{12}-\frac{14157}{4194304} k^{14}-\frac{2760615}{1073741824} k^{16}-\ldots\right) \\
& =\frac{\pi}{2}\left\{1-\sum_{n=1}^{\infty}\left[\frac{1 \cdot 3 \cdot . .(2 n-1)}{2 \cdot 4 \cdot \ldots \cdot 2 n}\right]^{2} \frac{k^{2 n}}{2 n-1}\right\}=\frac{\pi}{2}\left\{1-\sum_{n=1}^{\infty}\left[\frac{(2 n-1) ! !}{2^{n} n !}\right]^{2} \frac{k^{2 n}}{2 n-1}\right\} .
\end{aligned}
$$

Proceeding in the same manner, we get for the $1^{\text {st }}$ set (the most inaccurate) of approximate functions the expansions

$$
\begin{aligned}
& \mathrm{K}_{0}(k)=\frac{\pi}{2}\left(1+\frac{1}{4} k^{2}+\frac{9}{64} k^{4}+\frac{25}{256} k^{6}+\frac{1222}{16384} k^{8}+\ldots\right) ; \\
& \mathrm{E}_{0}(k)=\frac{\pi}{2}\left(1-\frac{1}{4} k^{2}-\frac{3}{64} k^{4}-\frac{5}{256} k^{6}-\frac{172}{16384} k^{8}-\ldots\right),
\end{aligned}
$$

for the $2^{\text {nd }}$ set being practically identical with the exact ones

$$
\begin{aligned}
& \mathrm{K}_{1}(k)=\frac{\pi}{2}\left(1+\frac{1}{4} k^{2}+\frac{9}{64} k^{4}+\frac{25}{256} k^{6}+\frac{1225}{16384} k^{8}+\frac{3969}{65536} k^{10}\right. \\
& \left.+\frac{53361}{1048576} k^{12}+\frac{184041}{4194304} k^{14}+\frac{41409222}{1073741824} k^{16}+\ldots\right) ; \\
& \mathrm{E}_{1}(k)=\frac{\pi}{2}\left(1-\frac{1}{4} k^{2}-\frac{3}{64} k^{4}-\frac{5}{256} k^{6}-\frac{175}{16384} k^{8}-\frac{441}{65536} k^{10}\right. \\
& \left.-\frac{4851}{1048576} k^{12}-\frac{14157}{4194304} k^{14}-\frac{2760606}{1073741824} k^{16}-\ldots\right) .
\end{aligned}
$$

The difference with respect to the expansions of the 
exact functions begins at the terms in $k^{8}$ for the $1^{\text {st }}$ set of approximation, and at the terms in $k^{16}$ for the $2^{\text {nd }}$ one. For the $1^{\text {st }}$ order derivatives of the exact functions we get $\frac{d \mathrm{~K}(k)}{d k}=\frac{\mathrm{E}(k)}{k\left(1-k^{2}\right)}-\frac{\mathrm{K}(k)}{k}=\frac{\pi}{4} k\left(1+\frac{9}{8} k^{2}+\frac{75}{64} k^{4}+\frac{1225}{1024} k^{6}\right.$ $\left.+\frac{19845}{16384} k^{8}+\frac{160083}{131072} k^{10}+\frac{1288287}{1048576} k^{12}+\frac{41409225}{33554432} k^{14}+\right)$ $=\frac{\pi}{4} \sum_{n=1}^{\infty}\left[\frac{1 \cdot 3 \cdot \ldots(2 n-1)}{2 \cdot 4 \cdot \ldots \cdot 2 n}\right]^{2} n k^{2 n-1}=\frac{\pi}{4} \sum_{n=1}^{\infty}\left[\frac{(2 n-1) ! !}{2^{n-1} n !}\right]^{2} n k^{2 n-1}$; $\frac{d \mathrm{E}(k)}{d k}=\frac{\mathrm{E}(k)-\mathrm{K}(k)}{k}=-\frac{\pi}{4} k\left(1+\frac{3}{8} k^{2}+\frac{15}{64} k^{4}+\frac{175}{1024} k^{6}\right.$ $\left.+\frac{2205}{16384} k^{8}+\frac{14553}{131072} k^{10}+\frac{99099}{1048576} k^{12}+\frac{2760615}{33554432} k^{14}+\ldots\right)$ $=-\frac{\pi}{4} \sum_{n=1}^{\infty}\left[\frac{1 \cdot 3 \cdot \ldots(2 n-1)}{2 \cdot 4 \cdot \ldots \cdot 2 n}\right]^{2} \frac{n k^{2 n-1}}{2 n-1}=-\frac{\pi}{4} \sum_{n=1}^{\infty}\left[\frac{(2 n-1) ! !}{2^{n-1} n !}\right]^{2} \frac{n k^{2 n-1}}{2 n-1}$. Applying the previous two exact relations and using the four definitions from chapter 2 one gets the expansions $\left[\frac{d \mathrm{~K}(k)}{d k}\right]_{0}=\frac{\pi}{4} k\left(1+\frac{9}{8} k^{2}+\frac{75}{64} k^{4}+\frac{1225.75}{1024} k^{6}+\ldots\right) ;$
$\left[\frac{d \mathrm{E}(k)}{d k}\right]_{0}=-\frac{\pi}{4} k\left(1+\frac{3}{8} k^{2}+\frac{15}{64} k^{4}+\frac{174.25}{1024} k^{6}+\ldots\right)$, for the $1^{\text {st }}$ set of approximate functions, and respectively $\left[\frac{d \mathrm{~K}(k)}{d k}\right]_{1}=\frac{\pi}{4} k\left(1+\frac{9}{8} k^{2}+\frac{75}{64} k^{4}+\frac{1225}{1024} k^{6}+\frac{19845}{16384} k^{8}\right.$ $\left.+\frac{160083}{131072} k^{10}+\frac{1288287}{1048576} k^{12}+\frac{41409226.125}{33554432} k^{14}+\ldots\right)$; $\left[\frac{d \mathrm{E}(k)}{d k}\right]_{1}=-\frac{\pi}{4} k\left(1+\frac{3}{8} k^{2}+\frac{15}{64} k^{4}+\frac{175}{1024} k^{6}+\frac{2205}{16384} k^{8}\right.$ $\left.+\frac{14553}{131072} k^{10}+\frac{99099}{1048576} k^{12}+\frac{2760614.25}{33554432} k^{14}+\ldots\right)$, for the $2^{\text {nd }}$ set of approximate functions. The difference with respect to the expansions of the $1^{\text {st }}$ order derivatives of the exact functions begins at the terms in $k^{7}$ for the $1^{\text {st }}$ set of approximation, and at the terms in $k^{15}$ for the $2^{\text {nd }}$ one, being much smaller than that for the expansions of the respective sets of approximate functions. One can also easily find the analytic expressions and series representations for the $2^{\text {nd }}$ derivatives of all $\mathrm{K}, \mathrm{K}_{0,1}, \mathrm{E}, \mathrm{E}_{0,1}$.

\section{Graphic comparison}

The variation curves of both Legendre complete elliptic integrals, as well as that of the two sets of newly proposed closed analytic functions are graphically represented in the comparative figures 1 and 2, all versus the angle $\theta$, expressed in sexagesimal degrees and given by $\theta=\sin ^{-1} k$. In both figures the exact functions $\mathrm{K}(k), \mathrm{E}(k)$ were represented by solid (continuous) black lines, the $1^{\text {st }}$ set of approximation $\mathrm{K}_{0}(k), \mathrm{E}_{0}(k)$ by dashed black lines, and the $2^{\text {nd }}$ set of approximation $\mathrm{K}_{1}(k), \mathrm{E}_{1}(k)$ by solid red lines, resp.

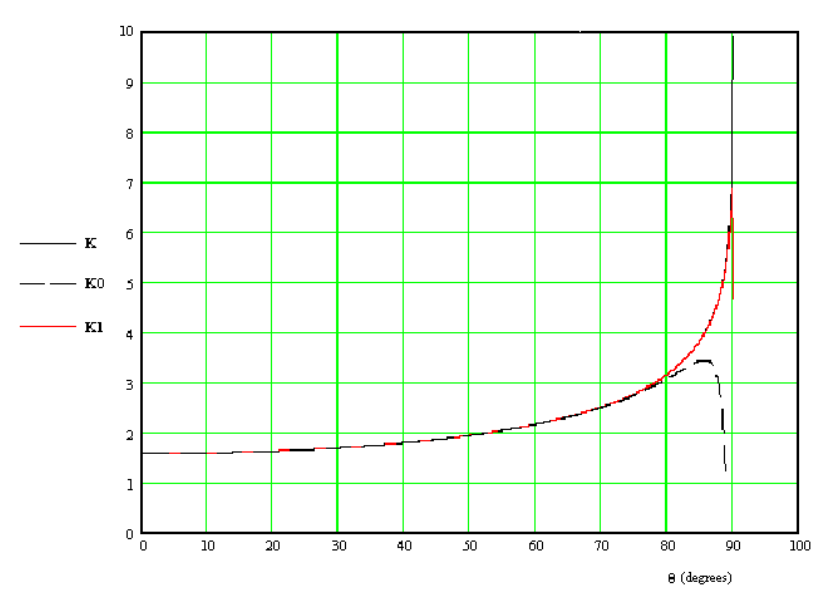

Fig. 1. Comparison of the Legendre complete elliptic integral $\mathrm{K}(k)$ with the closed analytic functions $\mathrm{K}_{0}(k), \mathrm{K}_{1}(k)$

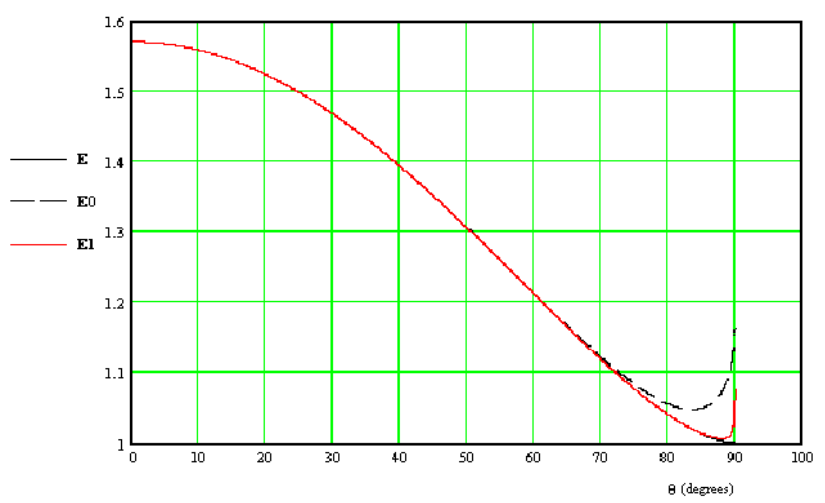

Fig. 2. Comparison of the Legendre complete elliptic integral $\mathrm{E}(k)$ with the closed analytic functions $\mathrm{E}_{0}(k), \mathrm{E}_{1}(k)$

\section{Conclusions}

As for their simplicity, the formulas in $k / k^{\prime}$ do not need any mathematical table (are purely algebraic). As for their accuracy, in current mathematical / technical applications, it must use the $1^{\text {st }}$ set until $\theta=70^{\circ} .5(k=0.94264)$ only, and for a better accuracy or a greater upper limit of the validity domain, to use the $2^{\text {nd }}$ set, but until $\theta=88^{\circ} .2(k=0.99951)$. Legendre complete elliptic integrals of the 1 st and 2nd kinds are very useful in Signal Processing and especially in the design of Digital Filters ( Legendre Digital Filters)

\section{Notes; other methods; future research}

With an appropriate reduction formula, every elliptic integral can be brought into a form that involves integrals over rational functions and the three Legendre canonical forms (of the $1^{\text {st }}, 2^{\text {nd }} \& 3^{\text {trd }}$ kind). Without the comparative tables 1 and 2, the errors table becoming so table 1, this work was published previously in a proceedings volume (scientific bulletin), in Romanian [8]. For the first English version of this work see [9]. Approximations for the complete elliptic integrals based on the trapezoidal-type numerical integration formulas discussed in [10], are developed in [11], [12] (a mixed numerical-analytic method). Newer formulas (using $\Gamma$ function-not an elementary, but a special one, likeK \& E, even if these formulas are the most accurate) are in [13], [14]; as stated in their abstracts, the works [9], [13] do not have the same goal. Notable special functions suitable for applying such an approximate method of calculation (like in [9]) are: $\mathrm{Si}(\mathrm{x})$; $\mathrm{Ci}(\mathrm{x})$; $\mathrm{Ei}(\mathrm{x})$; li(x). 


\section{References:}

[1] Legendre, A. M., Tables of the complete and incomplete elliptic integrals. Reissued (from tome II of Legendre's Traité des fonctions elliptiques, Paris, 1825) by K. Pearson, London, 1934.

[2] Heuman, C. A., Tables of complete elliptic integrals, J. Math. Physics, 20, pp. 127 - 206, 336, 1941; https:// onlinelibrary.wiley.com/doi/epdf/10.1002/sapm1941201127. [3] Hayashi, K., Tafeln der Besselschen, Theta-, Kugelund anderen Funktionen, Berlin, 1930; https://link.springer. com/content/pdf/bbm\%3A978-3-662-41028-8\%2F1.pdf.

[4] Hayashi, K., Tafeln für die Differenzenrechnung sowie für die Hyperbel-, Besselschen, elliptischen und anderen Funktionen, Berlin, 1933.

[5] Jahnke, E., Emde, F., Tables of Functions with Formulae and Curves, Dover Publications, New York, 1943; Fourth Edition, 1945; (translated into Russian: Е. Янке и Ф. Эмде, Таблищь функии с формулами и кривыми, Физматгиз, Москва - Ленинград, 1959;) [6] Jahnke, E., Emde, F., Lösch, F., Tafeln höherer Funktionen, sechste Auflage. Neubearbeitet von F. Lösch, B. G. Teubner Verlagsgesellschaft, Stuttgart, 1960, 1961; https://doi.org/10.1002/zamm.19610410619; (translated into Russian: Е. Янке, Ф. Эмде, Ф. Лёш, Специальные функиии - формуль, графики, таблиць, Наука, Москва, 1964; $\quad$ https://ikfia.ysn.ru/wpcontent/uploads/2018/01/JankeEmdeLyosh1964ru.pdf). [7] Gradshteyn, I. S., Ryzhik, I. M., Table of Integrals, Series, and Products. Fourth Edition Prepared by Yu. V. Geronimus / M. Yu. Tseytlin, Academic Press, New York, London, 1965; Translated from Russian by Scripta Technica, Inc.; Seventh Edition, 2007; Eds.: A. Jeffrey, D. Zwillinger; http://fisica.ciens.ucv.ve/ svincenz/TISPISGIMR.pdf. there are Russian, German, Polish, English \& Japanese eds.; [8] Selescu, R., Formule analitice închise pentru aproximarea integralelor eliptice complete de speța întâia şi a doua ale lui Legendre, Buletinul Ştiințific al Sesiunii Naționale de Comunicări Ştiințifice, Academia Forțelor Aeriene "Henri Coandă" \& Centrul Regional pentru Managementul Resurselor de Apărare, Editura Academiei Forțelor Aeriene "Henri Coandă", Braşov, 1 -2 Nov. 2002; Vol. MATEMATICA - INFORMATICA, Anul III, Nr. 2 (14), (ISSN 1453-0139), pp. 37 - 44; (in Romanian).

[9] Selescu, R., Closed Analytic Formulas for the Approximation of the Legendre Complete Elliptic Integrals of the First and Second Kinds, International Journal of Mathematical and Computational Methods, Vol. 21, pp. 49 - 55, 21 May 2021;

http://www.iaras.org/iaras/journals/ijmcm.

[10] Luke, Y. L., Simple formulas for the evaluation of some higher transcendental functions, J. Math. Physics, v. 34, pp. 298 - 307, 1956, MR 17, \# 1138. [11] Luke, Y. L., Approximations for Elliptic Integrals, Math. Comp., Vol. 22, No. 103 (Jul. 1968), pp. 627-634, MR 17, \# 2412; American Mathematical Society; https:// ams.org/journals/mcom/1968-22-103/S0225-57181968-0226825-3/S0025-5718-1968-0226825-3.pdf. [12] Luke, Y. L., Further Approximation for Elliptic Integrals, Math. Comp., Vol. 24, No. 109 (Jan. 1970), pp. 191 - 198, American Mathematical Society; htpps:// ams.org/journals/mcom/1970-24-109/S0025-57191970-0258243-5/S0025-5719-1970-0258243-5.pdf. [13] Bagis, N., Formulas for the approximation of the complete Elliptic Integrals,

https://arxiv.org/abs/1104.4798v1 [math.GM], 6 pages (pp. 1 -6), 25 April 2011, Cornell University, preprint. [14] Bagis, N., Formulas for the Approximation of the Complete Elliptic Integrals, International Mathematical Forum, Vol. 7, no, 55, pp. 2719 - 2725, 2012; http://www.m-hikari.com/imf/imf-2012/53-56-2012/ bagisIMF53-56-2012.pdf; (supersedes [13]).

\section{Creative Commons Attribution License 4.0 (Attribution 4.0 International, CC BY 4.0)}

This article is published under the terms of the Creative Commons Attribution License 4.0

https://creativecommons.org/licenses/by/4.0/deed.en US 